\title{
Clouds Handling for Planning of Optical Space Missions
}

\author{
B. Grishechkin ${ }^{1}$, A. Braun ${ }^{2}$, M. Wickler ${ }^{3}$ \\ German Space Operation Center, Munich, Bavaria, 82234, Germany
}

\begin{abstract}
Every space mission which uses optical band, e.g. ground-satellite/satellite-ground laser telecommunication, optical earth observation, on-ground optical space debris tracking system, is drastically affected by clouds in the troposphere of the Earth. Mission planning group of the German Space Operations Center (GSOC), DLR is investigating the possibility of achieving the maximum performance of future optical space missions. Planning of an optical space mission has to involve clouds cover data. Relevant long-term past data from ECMWF (UK) and short-term predicted data from NOAA (USA) in standard GRIB format were used for this work. Short-term predicted future data are helpful for operational planning of an on-ground station and the relevant satellite. Long-term past data can be used to design an optimal network of on-ground stations and to assess the performance of the whole mission. Several methods of time-interpolation of the clouds cover data were compared with and without using wind information, a corresponding software tool was developed.
\end{abstract}

\section{Introduction}

A important part of each mission operated by GSOC, especially earth observation missions, is a Mission Planning System. Its first task is collecting and analyzing of information, availabilities and requirements ${ }^{1}$ that are relevant to the on-board operations.

For future missions which use optical band, e.g. optical Earth remote sensing EnMAP mission or a laser telecommunication mission, a new component of MPS which reads and processes relevant meteorological data have to be developed, it is called further cloud information software (CIS). The common format for meteorological data is general standard format $\mathrm{GRIB}^{2}$. Files of this format can include values of a couple of hundreds meteorological parameters. The most useful parameters for CIS seem to be total cloud cover (TCC) and wind speed. Each GRIB file is divided into GRIB-messages, which usually have the same structure and simplified contain values of a meteorological parameter for the same area but for different time. Such meteorological data with cloud information (CI) is delivered by different companies, among them e.g. DWD (Germany), ECMWF (UK), NOAA (USA), Weatheroffice (Canada). While developing CIS it was taken into account that the mentioned standard has already changed several times, there are already at least two incompatible versions of the format (GRIBv1, GRIBv2) and that it can be changed again. Under processing of the mentioned files a time- and spatial-interpolation is understood as MPS needs clouds cover for a particular time and place which is generally not explicitly given in the CI.

\section{Development of CIS}

As the output of CIS is a value of total cloud cover and the necessary input is:

- date and time

- geographical coordinate

- a corresponding GRIB file

\footnotetext{
${ }^{1}$ Scientific fellow, Mission planning group in GSOC, DLR, Münchner Strasse 20.

${ }^{2}$ Group leader, Mission planning group in GSOC, DLR, Münchner Strasse 20.

${ }^{3}$ Chief of department, Earth observation department in GSOC, DLR, Münchner Strasse 20.
} 
the structure of CIS can be represented as in Fig. 1.

As one can see in Fig. 1 there is also an XML schema file as a virtual input of the CIS. This is not an operational input for the software. It is used only during the phase of compiling and building of CIS. The XML schema file describes the used GRIB format (v1 or v2) in extensible markup language and serves as a translation of the human understandable standard into a computer understandable description. This file can be updated as soon as the standard is changed and editing of such a file is much easier than editing of the source codes of the CIS.

One should mention a special peculiarity of the described XML schema. Apparently using of XML schemas to describe intersoftware interfaces is nothing new if the interaction is fulfilled by meaning of standardized text XML data files. In the case of GRIB

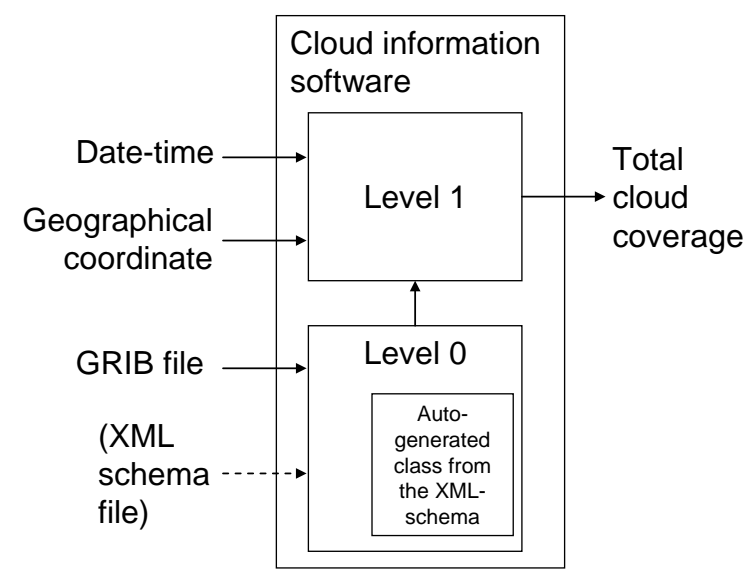

Figure 1. The structure of CIS.

format not only the data files are binary but also they use its own compression of data contained in some sections. Therefore the automatically generated class from the XML schema is just a part of the so called level 0 component of CIS, it implements the developed rules of interpreting the standardized XML schema description into binary file access methods. Level 1 component of CIS implements the data unpacking methods and the target algorithms of time- and spatial interpolation, fulfils coordinate system conversion if needed.

For assessment of the interpolation algorithms and for general debugging purposes a GUI was developed.

\section{Time interpolation in CIS}

As one fulfills an optical mission planning one needs to know the cloud cover at particular coordinates at a particular time, and the existing CI is usually created for a common consumer with some coordinate and time step, one should interpolate the data spatially and in time. The only difficulty of spatial interpolation is that the coordinates of the given grid is not linearly dependent on the on-surface distances between the given coordinate grid nodes. As for interpolation in time apparently there can be two principally different types of interpolation of CI. The first type uses wind information and the second type uses only CI to calculate its intermediate values. The task of generating the CI with a less step in time and with less spatial step is not considered here because it is a complex task of meteorological models which has at least tens of parameters an need too much computing resources. The following is a description and comparison of developed methods of interpolation of both type (using and not using wind information).

\section{A. Used GRIB files}

GRIB file N1 is from NOAA and was downloaded for free from its internet server by software called zyGrib and contained the following predicted data:

- period: 11.04.2011 - 13.04.2011;

- types of data: "Wind: 10m" (u- and v- components), "Cloud cover: atm";

- time resolution: 6 hours;

- 8 GRIB-messages per each type of data;

- area: $80^{\circ} \mathrm{S} 170^{\circ} \mathrm{W}-80^{\circ} \mathrm{N} 170^{\circ} \mathrm{E}$;

- spatial resolution: $2^{\circ} \mathrm{x} 2^{\circ}$.

GRIB file N2 is from ECMWF and was bought among a set of similar files for a period of several years:

- period: 26.10.1996 - 31.12.1996;

- types of data: "Total cloud cover";

- time resolution: 6 hours;

- 268 GRIB-messages;

- area: the entire Earth;

- spatial resolution: $0.5^{\circ} \mathrm{x} 0.5^{\circ}$.

The data in used files from ECMWF do not contain wind information therefore only data from NOAA could be used to investigate and assess the quality of interpolation based on the wind information. 


\section{B. Simple wind-driven interpolation}

The speed of the cloud in each node of the coordinate grid is assumed to be equal to the wind speed given at this node but taken from the next (in terms of time +6 hours) GRIB-message. If any such node after being "moved" by wind appears inside the tolerance area of another node the values of cloud cover for these nodes are summed, see Fig. 2. The size of the tolerance area (TOL) was taken from 0,4 to 1,5 of the coordinate grid step.

This type of modeling can produce locally cloudless areas with sharp edges, see some highlighted areas in Fig. 3, what indeed depends on the method of "summing" of cloud covers but seems like a too rough modeling of clouds' movement. As the physical meaning of the cloud cover value in GRIB files is not standardized several types of "summing" of cloud cover were checked:

1) Statistical. The cloud cover is "summed" like a random noise, see Eq. 1, where $T C C_{\text {moved }_{i}}$ is cloud cover value of a pixel moved by the wind and which came in to the tolerance area for current pixel, $n$ is the number of values being "summed" (for mentioned file N1 $n$ was about from 0 to 14 ).

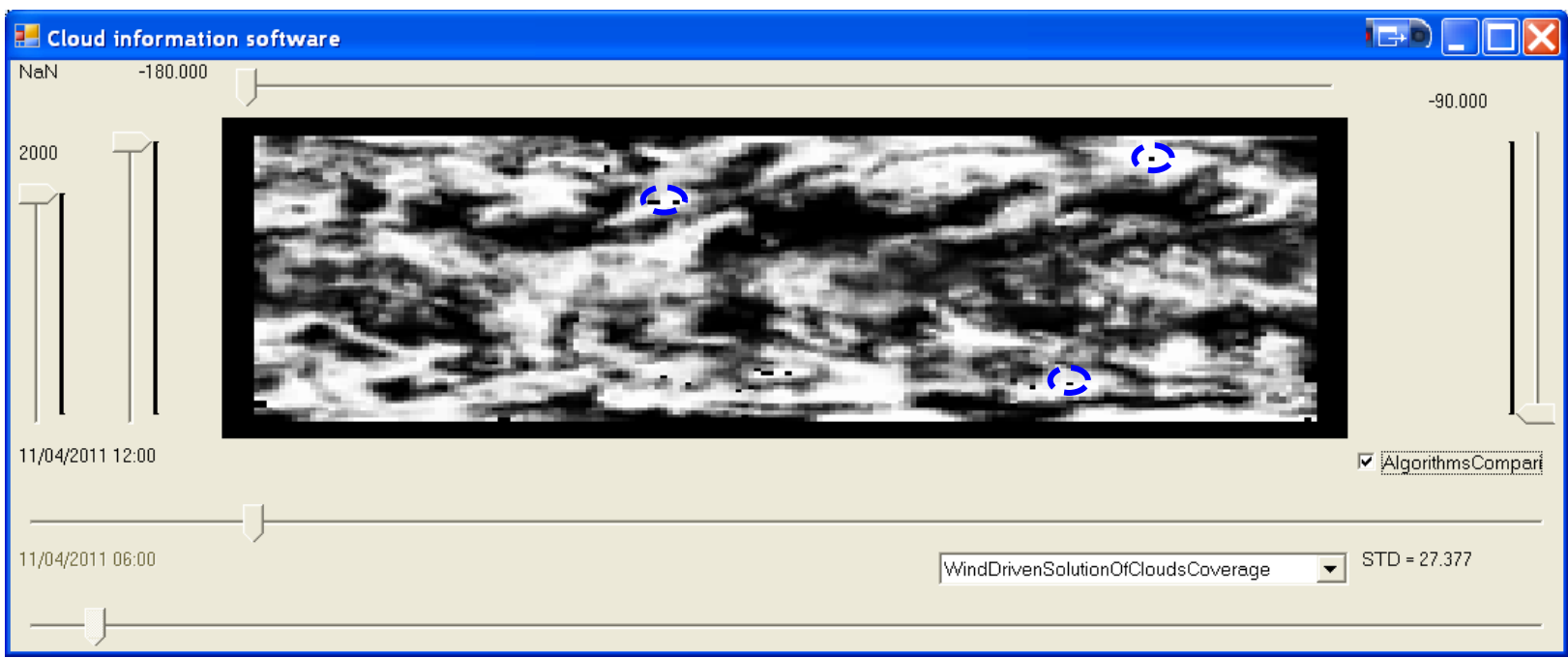

Figure 3. An apparent disadvantage of "wind-driven" time interpolation

$$
\text { TCC }_{\text {model }_{i}}=\sqrt{\frac{1}{n} \sum_{i=1}^{n} T^{2} C_{\text {moved }_{i}}^{2}}
$$

2) Averaging. The result cloud cover is calculated a mean value of the values to be "summed".

3) Optical. The cloud cover is calculated like opacity of several layers of light barriers, see Eq. 2,

$$
\text { TCC }_{\text {model }_{i}}=100 \times\left(1-\prod_{i=1}^{n}\left(1-\frac{T}{10 C_{\text {moved }}}\right)\right)
$$

4) Maximum value. The result cloud cover is taken equal to the maximum cloud cover of the pixels came to the tolerance area of current pixel. 
The difference of which GRIB-message (previous, current, next) to use for wind information is almost negligible: we noticed that the standard deviation when taking wind from these different GRIB-messages changes less than by $5 \%$.

\section{Cell stretching interpolation}

Each node of the coordinate grid is considered as belonging to a rectangular cell which is located down-right under the node. In the beginning the cloud cover is known in each cell, i.e. it is read from the GRIB-file. Each node of the grid is moved with the speed of the wind relevant to this node, after that we get new cells, distorted in

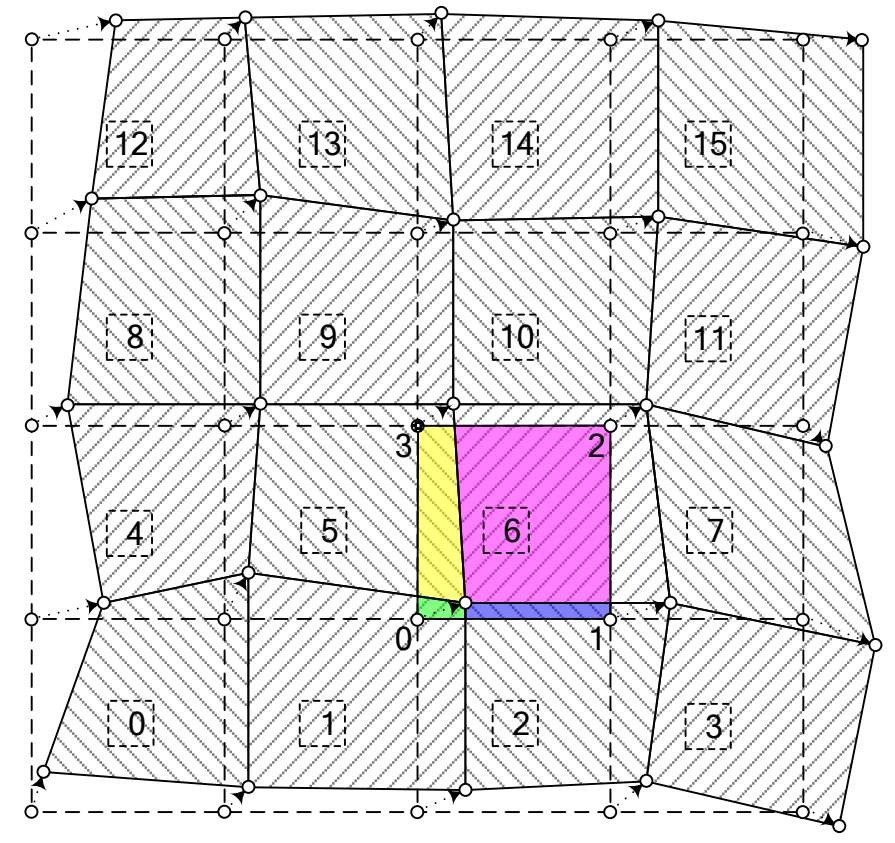

Figure 4. Original cells, distorted cells, numbering of nodes and cells

weight for each value is proportional to the shared area for the intersected cells. In Fig. 4 these shared areas of the cell relevant to the hatched node are highlighted with different colors.

When calculating the shared area for the intersected cells and the area of the original cells each area is perceived as a polygon which is divided into spherical triangles, see Fig. 5. Area $S$ of each spherical triangles is calculated using Eq. (4), where the radius-vectors $O A, O B, O C$ for a triangle ABC are shown in Fig. 6, and $r=6378137 \mathrm{~m}$ - the radius of the Earth. comparison with original rectangular cells. The cloud cover in each distorted cell is assumed to be equal to the cloud cover in the appropriate original cell. Then the new cloud cover of each cell in the original grid is calculated. In Fig. 4 it is shown that the new total cover in each cell should be calculated as a weighted sum of the values of cloud coverage of the nearest cells an the original cell, see Eq. 3.

$$
\text { TCC }_{\text {model }_{i}}=\sum_{i=1}^{9}\left(\frac{\text { PolygonArea }_{i}}{\text { CellArea }_{\text {lat }, \text { lon }}} \cdot \text { TCC }_{\text {read }_{i}}\right)
$$

For the cell 6 presented in Fig. 4 four values should be weighted. In general the amount of the values to be weighted for each cell is different and depends on the wind. The

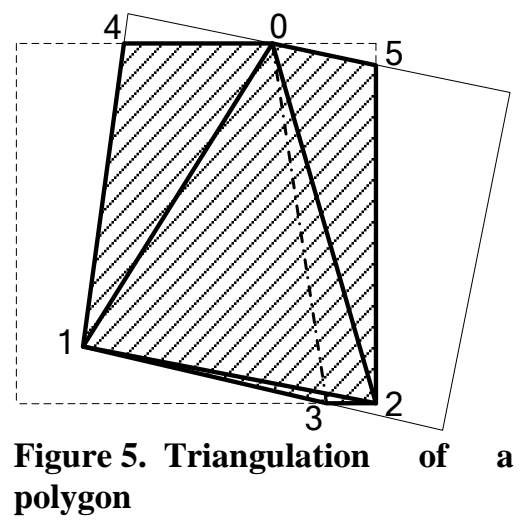

$$
\begin{aligned}
S= & \frac{r^{2}}{2}\left(\arccos \left(-\frac{(\overline{O C} \times \overline{O A}) \cdot(\overline{O A} \times \overline{O B})}{|\overline{O C} \times \overline{O A}| \cdot|\overline{O A} \times \overline{O B}|}\right)+\right. \\
& \left.+\arccos \left(-\frac{(\overline{O A} \times \overline{O B}) \cdot(\overline{O B} \times \overline{O C})}{|\overline{O A} \times \overline{O B}| \cdot|\overline{O B} \times \overline{O C}|}\right)+\arccos \left(-\frac{(\overline{O B} \times \overline{O C}) \cdot(\overline{O C} \times \overline{O A})}{|\overline{O B} \times \overline{O C}| \cdot|\overline{O C} \times \overline{O A}|}\right)-\pi\right)
\end{aligned}
$$




\section{Linear interpolation}

Linear interpolation is evidently the simplest method which comes to mind. Its idea is that a modeled value of cloud cover for each node is calculated based on two values of the cloud cover for this node: the nearest previous and the nearest next value in time which is read from the GRIB file. So this value does not depend on the cloud cover of the surrounding nodes and does not take wind information into account at all.

\section{E. Comparison of interpolation methods}

The quality of clouds' movement modeling described above was calculated as standard deviation of the modeled cloud cover from the cloud cover originally read from the GRIB file for the same time,

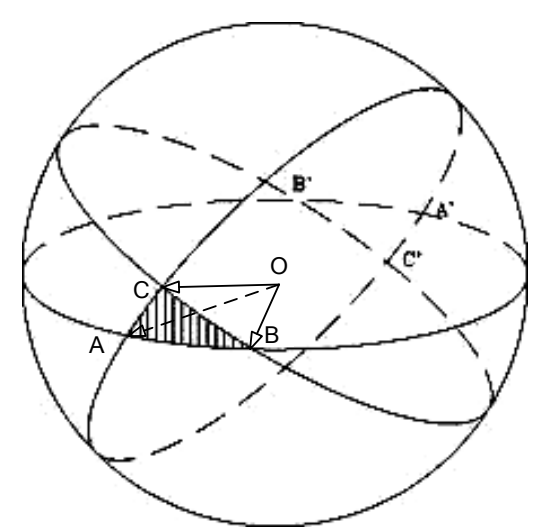

Figure 6. Spherical triangle ABC on the Earth surface see Eq. (5), where $m$ is the number of coordinate grid nodes given in the GRIB file (for the file $\mathrm{N} 1 \mathrm{~m}=81 \times 171=$ 13851), $T C C_{\text {model }_{i}}$ modeled total cloud cover in the $i$-node given in $\%$ units, $T C C_{\text {read }_{i}}$ total cloud cover in $\%$ units read from file for the $i$-node. Therefore for quality assessment CIS modeled a 6-hour forecast based on the previous and depending on the algorithm on the next GRIB-message and therefore it was possible to compare one or two message less than there were in the GRIB file.

$$
S T D=\sqrt{\frac{1}{m} \sum_{i=1}^{m}\left(\text { TCC }_{\text {model }_{i}}-\text { TCC }_{\text {read }_{i}}\right)^{2}}
$$

The appropriateness of using standard deviation was checked by analyzing of the distribution of modeling error. For all presented methods of interpolation, independently on the particular GRIB-message, the distribution of modeling error was close to Gaussian with zero mean, see Fig. 7-9, that allowed us to use normal standard deviation as a quality criteria for the modeling.

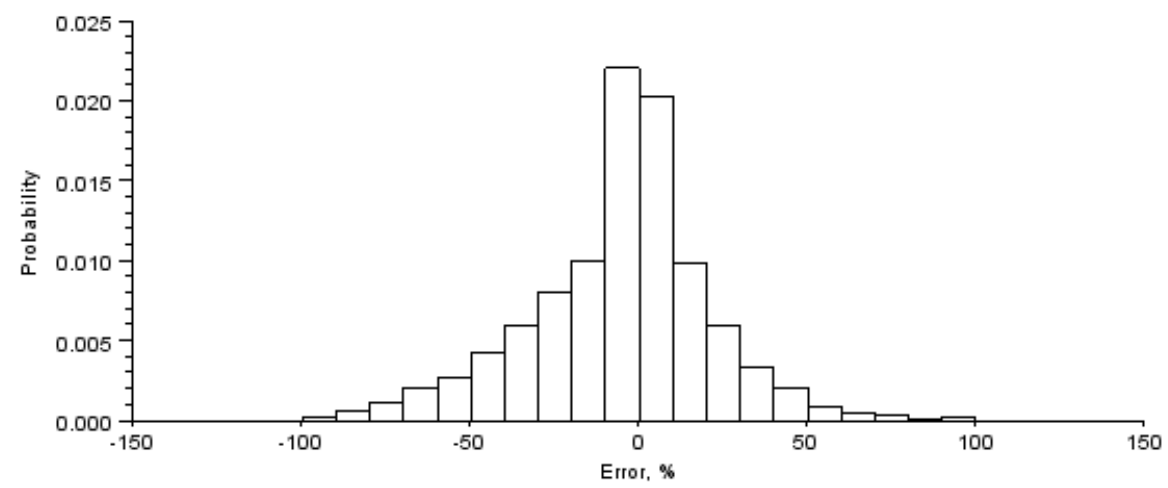

Figure 7. Distribution of "wind-driven modeling" error, "statistical" method, TOL = 1,1, modeling for 11.04.2011 12:00 


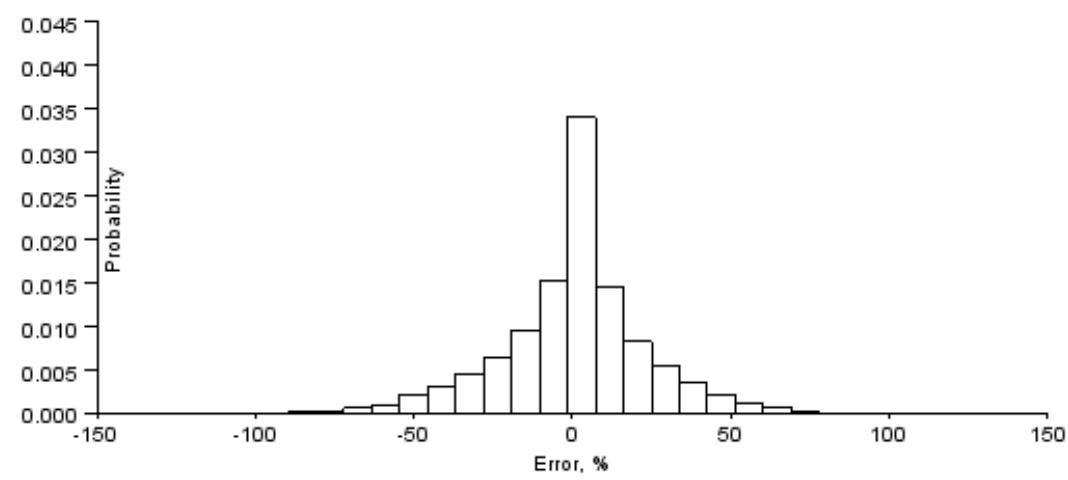

Figure 8. Distribution of modeling error of the "cell stretching" algorithm, modeling for 11.04.2011 12:00

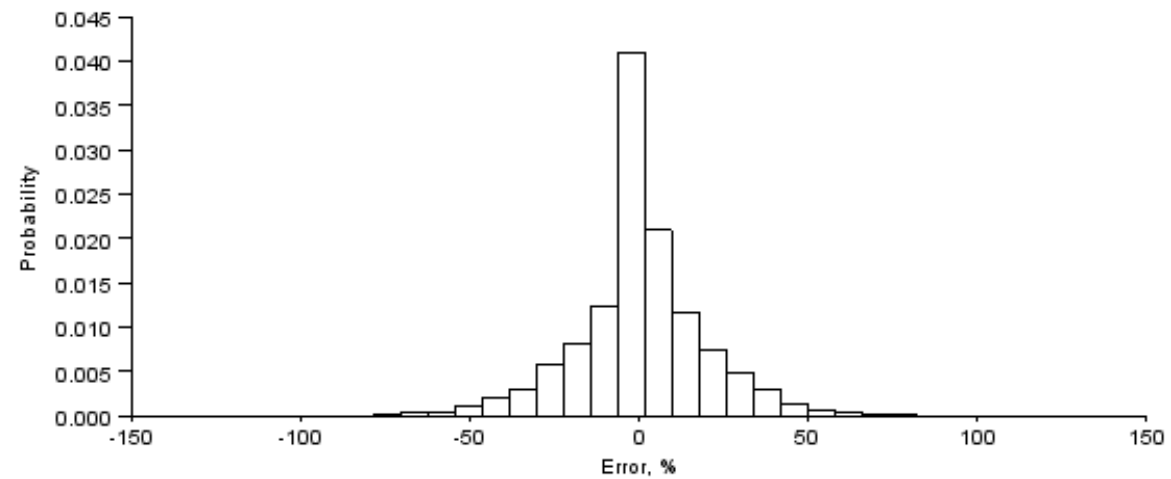

Figure 9. Distribution of "simple linear time interpolation of clouds" modeling error, modeling for 11.04.2011 12:00

The comparison results for each type of interpolation are presented in Table 1. Our results show that for the mentioned available meteorological data it is more precisely to use linear interpolation without using wind information.

Table 1. Standard deviation of modeled by CIS cloud cover from given cloud cover

\begin{tabular}{|l|c|c|}
\hline \multicolumn{1}{|c|}{ Algorithm } & Min STD, \% & Max STD, \% \\
\hline Linear interpolation & 18,327 & 18,761 \\
\hline Cell stretching & 22,205 & 23,261 \\
\hline Simple wind-driven interpolation (TOL = 1,1): & & \\
1) statistical & 23,851 & 24,754 \\
2) averaging & 23,406 & 24,216 \\
3) optical & 39,916 & 41,512 \\
4) maximum method & 33,230 & 34,599 \\
\hline
\end{tabular}

\section{F. Remarks about wind information}

While analyzing interpolation with using wind information the wind information itself was manually observed as a matrix of arrows on a map displayed by the zyGrib software and compared with the intermediate CI calculated by CIS. It was clearly noticed that in some areas the wind blew from east to west (and the modeled clouds' movement direction in CIS was the same) but the according to the original CI read from the GRIB file the clouds were moving in the opposite direction. This is apparently possible if the cloud movement does not depend on the given wind, what is pretty probable because the given wind was for the attitude of only $10 \mathrm{~m}$ above the surface. This fact can also be the reason why the linear time interpolation of clouds without using wind information gave better result. 
This also could be possible in the case of wrong usage of the wind direction but this idea was also checked. In the "FM 92-XI ext. GRIB edition 1" standard it is not specified if the wind blows to the given direction or from the given direction. In the "FM 92 GRIB v2" standard the clear "from which blowing" term is used. When the term "from which blowing" instead of "to which it blows" was applied to the data from the mentioned file the modeling result was even worse though the clouds' movement in the mentioned areas looked more adequate.

There is also some information ${ }^{3}$ that there is a very little dependency of the clouds' movement on the

atmospheric flow (wind) especially in mountain areas. A possible solution of the problem could be to use so-called cloud motion wind which is not actually meteorologically consistent atmospheric flow.

\section{Preoperational use of CIS}

By using some past long-term cloud information and particular use case of a future mission one can assess an overall efficiency of the mission. This can be very helpful while planning a new optical mission like telescope observations or on-ground optical terminals for satellite telecommunication. One can calculate some visibility statistics to get some overview ${ }^{4}$, to assess some preliminary parameters for scheduling or to choose an optimal position for a new building with a mission component.

\section{Conclusion}

Having some experience on working with available cloud and wind information in GRIB standard format files we would like to notice that it is recommended if applicable to discuss some standard parameters of the format before ordering the data, e.g. compression type, coordinate grid type. Though the standard is pretty large and informative it is also necessary to ask the provider for additional description which meteorological data is in there, which physical meaning it has and how it should be used for your needs.

As the continuation of the development of the presented CSI we see implementing of a solution of optimal positioning of on-ground stations for an optical communication satellite mission. 


\section{Appendix A \\ Acronym List}

$\begin{array}{ll}\text { GRIB } & \text { Gridded binary } \\ \text { CI } & \text { Cloud information } \\ \text { CIS } & \text { Cloud information software } \\ \text { MPS } & \text { Mission planning system } \\ \text { TCC } & \text { Total cloud cover } \\ \text { XML } & \text { Extensible markup language }\end{array}$

\section{References}

${ }^{1}$ Wickler, M., “A Mission Planning Concept and Mission Planning System for Future Manned Space Missions,” Proceedings of SpaceOps 1994 [online publication], URL: http://www.op.dlr.de/SpaceOps/spops94/wickler/wickler.htm [cited 26 April 2012].

${ }^{2}$ WMO PUBLICATION No. 306 - MANUAL ON CODES, Volume I.2.

${ }^{3}$ CineSat, "Frequently asked questions about CineSat", URL: http://www.cinesat.com/csfaq.htm\#NWPWinds [cited 26 April 2012].

${ }^{4}$ Kenta Ogawa, Tsuneo Matsunaga, Satoru Yamamoto, Osamu Kashimura, Tetsushi Tachikawa, Akira Iwasaki, Satoshi Tsuchida, Jun Tanii and Shuichi Rokugawa, "Operation planning for Japanese future hyperspectral and multispectral senor: HISUI and usage of cloud climate data", Sensors, Systems, and Next-Generation Satellites XV Proceeding, edited by Roland Meynart, Steven P. Neeck, Haruhisa Shimoda, Vol. 8176, 817623, 2011. 